\section{O35 (continued)}

School of Humanities and Social Sciences, University of Cambridge; Mei-Yen Chan, SRD, PhD, School of Medicine, Nazarbayev University, Kazakhstan, NNEdPro Global Centre for Nutrition and Health

Background: The COVID-19 pandemic disrupted agrifood and health systems, increasing the risk of food insecurity, malnutrition, and related health problems.

Objective: To develop a global pandemic impact picture around agri-food and health systems.

Study Design, Setting, Participants: Cross-sectional web-based survey with closed- and open-ended questions. Food, nutrition, and health researchers/practitioners from an international network were recruited as representatives of populations they serve. Two reminders to complete the survey were sent.

Measurable Outcome/Analysis: Groups vulnerable to food insecurity and government actions were mapped, along with the impact of the pandemic on food production, distribution, and access, and offer of nutrition services. Descriptive statistics and content analysis summarized the data.

Results: Thirty individuals from Africa, America, Asia, Oceania, and Europe responded (11.85\%). Most were from nutrition and dietetics (43.3\%) or medicine (26.7\%), working in research $(50 \%)$ and with $>10$ years experience (62.1\%). Informal/temporary workers (83.3\%), older adults with chronic diseases (73.3\%) and children eligible for school meals (53.3\%) were found to be vulnerable to food insecurity. Commonly cited government actions were support for hand sanitation (53.3\%), assistance to school-aged children (46.7\%) and direct food provision (43.3\%). About $50 \%$ saw community-led actions as important solutions. Only $16.7 \%$ mentioned remote delivery of nutrition services in primary care. Open-ended questions revealed that economic shocks, reduced investment, lack of staff/staff illnesses, transit restrictions, markets/stores closure or panic buying contributed to food production/distribution constraints. Reduced food availability, with unemployment/ reduced purchasing power, increased food costs, lack of food security programmes or food emergency services contributed to food/nutrition insecurity. Nutrition services were reduced, suspended, or deprioritised.

Conclusion: Several factors contributed to agri-food systems disruption and various government actions were implemented globally. Nutrition services offered in the healthcare context deserves further exploration. As the pandemic continue this provide a blueprint for a nutrition education/awareness programme to mitigate those risks based on knowledge gaps in policy and practice.

Funding: NNEdPro Global Centre for Nutrition and Health.

\section{College Students Cope to Achieve Food Security During the COVID-19 Pandemic}

Miriam Manboard, BS, Texas State University; Lesli BiedigerFriedman, PhD, MPH, RDN, LB47@txstate.edu, Texas State University, 600 University Dr, San Marcos, TX, 78666; Hannah Thornton, MS, RD, LD, Texas State University;
Kelsey Walling, BS, Texas State University; Victoria Padilla, $B S$, Texas State University

Background: Feeding America estimates an additional 13.2 million people experienced food insecurity in 2020 as a result of the COVID-19 pandemic. College students have higher risk given their transitionary life stage and lack of access to enablers of food security, including federal food assistance programs. This project investigates interpersonal and community-level factors of the social ecological model that affect food choices, self-efficacy, and coping mechanisms among food insecure college students during the pandemic.

Objective: Describe facilitators and barriers to food security among college students during the COVID-19 pandemic.

Study Design, Setting, Participants: After IRB approval was obtained, participants $(\mathrm{n}=18)$ were interviewed via a video-conferencing platform to investigate interpersonal coping mechanisms related to food security. Inclusion criteria encompassed current enrollment, participation in the on-campus food pantry, and age less than 30 years. Interviews focused on students' food acquisition and utilization, experiences with food insecurity before and during the pandemic, and feelings of stress.

Measurable Outcomes/Analysis: Interviews were recorded, transcribed, and themed using a 2-coder method.

Results: Students identified lack of knowledge about food assistance programs, confusion over food assistance qualification, and lack of transportation to food assistance sites as perceived barriers to improving food security during the pandemic. Primary coping mechanisms and facilitators to obtaining food were food pantry utilization, social support through food sharing, participation in the Supplement Nutrition Assistance Program (SNAP), and engagement with community support programs.

Conclusion: COVID-19 has further exacerbated college students' vulnerability to food insecurity. College students have limited access to SNAP and rely on interpersonal relationships and community resources to maintain food security. Findings describe unique coping strategies to access food and identify strategies to improve college student food security during the pandemic.

Funding: United Way for Greater Austin.

\section{Research Methods \& Food Systems}

\section{Fruit and Vegetable Preparation Changes During and After Cost-Offset Community Supported Agriculture and Nutrition Education}

Grace Marshall, MHS, gam263@cornell.edu, Cornell University, 618 Tower Rd, Ithaca, NY, 14850; Karla Hanson, PhD, Cornell University; Jennifer Garner, PhD, RD, The Ohio State University; Alice Ammerman, DPH, University of North Carolina at Chapel Hill; Stephanie Jilcott Pitts, PhD, East Carolina University; Jane Kolodinsky, $P h D$, University of Vermont; Marilyn Sitaker, MPH, The 


\section{O37 (continued)}

Evergreen State College; Rebecca Seguin, $P h D, R D$, Texas A\&M Agrilife Research

Background: Most adults and children in the US do not consume recommended levels of fruit and vegetables (FV). Providing subsidized or cost-offset community supported agriculture (CO-CSA) alongside nutrition education is 1 approach to increase FV intake among rural, low-income families.

Objective: This analysis assessed change in the frequency and method of FV preparation during and after a CO-CSA plus nutrition education intervention.

Study Design, Setting, Participants: Participants were caregivers in low-income households $(<185 \% \mathrm{FPL})$ living in rural areas of 4 U.S. states $(n=148)$. Half-price weekly summer CSA shares of farm produce and 9 seasonal nutrition education lessons were provided. Attendance was recorded at lessons and other data were collected via online surveys at baseline, 1 season later, and 1 year later.

Measurable Outcome/Analysis: Outcomes included monthly frequency of preparing 9 different, seasonal FV for children's snacks and 6 different vegetables for dinner with preparation methods for each. Repeated measures ANOVA and pairwise comparisons with Bonferroni correction tested change in FV preparation frequency over time at $95 \%$ confidence. Moderation analysis assessed whether changes in FV preparation frequency varied by lesson attendance.

Results: At baseline, caregivers prepared fruit for snacks and vegetables for dinner almost every day, and vegetables for snacks every other day. Preparation frequency for total snack fruit, celery, cucumber, peppers, other vegetables, total snack vegetables, lettuce, cabbage, greens, squash, and total dinner vegetables all increased during the intervention; and, increases in vegetables for both snacks (celery, cucumber, and total) and dinner (greens and total) were maintained oneyear later. At baseline, the majority of participants $(>70 \%)$ reported using healthy methods to prepare vegetables, and no changes were observed over time. Observed increases in FV preparation frequency were not moderated by lesson attendance.

Conclusion: CO-CSA plus education was associated with increases in FV preparation frequency, increases in vegetable preparation were maintained 1 year later, but improvements were not associated with attending nutrition education.

Funding: NIFA.

\section{How Seafood Says "Sustainable": A Content Analysis of Retail Package Labels}

Lauren B. Errickson, MS, lauren.errickson@rutgers.edu, Rutgers, the State University of New Jersey, 88 Lipman Dr, Room 316, New Brunswick, NJ, 08901; Helena Silva Horikawa, Rutgers, the State University of New Jersey; William K. Hallman, PhD, Rutgers, the State University of New Jersey; Mark Hanna, AS, Rutgers, the State University of New Jersey
Background: When harvested or raised within sustainable systems, products of domestic fisheries and aquaculture offer healthy, affordable seafood choices with minimal environmental impact that can help Americans meet dietary guidelines for seafood intake. However, some consumers hesitate to purchase seafood products, associating them with negative environmental impact, and adherence to intake recommendations remains low. Consistent sustainability labeling on seafood products could better signal environmental responsibility and encourage consumers toward increased intake.

Objective: To characterize label attributes indicative of sustainability on seafood packages.

Study Design, Setting, Participants: A content analysis of seafood package labels was conducted using the Label Insight product database. Characterized attributes included 320 terms and certifications documented on packages. From over 400,000 food products, 2,200 seafood packages were selected for inclusion based on type: salmon $(n=730)$, shrimp $(n=1,387)$, and oysters $(n=83)$. Mixed dishes were excluded.

Measurable Outcome/Analysis: Four trained coders characterized label attributes according to a priori themes, including sustainability. Total usage frequencies of sustainability-related labels, and frequencies of specific terms and certifications, were determined for each seafood type. Pearson chi-square analyses were conducted to evaluate statistical associations between seafood type and sustainability labeling practices.

Results: Thirty-eight attributes $(11.9 \%)$ were coded as sustainability labels. Overall, more salmon (47.9\%) and shrimp (47.6\%) packages displayed sustainability labels than did oysters $(26.5 \%), \quad\left(\chi^{2} \quad(2, N=2,200)=14.443\right.$, $P=0.001)$. Specific terms used varied by seafood type. Salmon (41.6\%) and oyster (26.5\%) packages displaying sustainability labels most frequently utilized unstandardized "environmental" terms, whereas shrimp packages (36\%) most often displayed a third-party sustainability certification label. Lesser-used terms included planet friendly, responsibly produced, and responsibly caught.

Conclusion: While encouraging that nearly half the evaluated salmon and shrimp packages reference sustainability, use of terminology and certifications across and within seafood types is inconsistent. Future studies might investigate whether more frequent use of standardized sustainability labels measurably increases seafood purchase and consumption.

Funding: USDA.

\section{Validating the Campus Food Aid Self- Assessment Tool (C-FAST)}

Sara Rains, BS, sar0016@auburn.edu, Hunger Solutions Institute at Auburn University, 210 Spidle Hall, Auburn, AL, 36849; Alicia Powers, PhD, MS, BS, Hunger Solutions Institute at Auburn University

Background: Recent studies have brought attention to college student food insecurity (FI), which is estimated to 